\title{
HYPERCONVEXITY AND NONEXPANSIVE MULTIFUNCTIONS
}

\author{
ROBERT SINE
}

\begin{abstract}
It is shown that a ball intersection valued nonexpansive multifunction on a hyperconvex space admits a nonexpansive point valued selection. This implies fixed point theorems for such multifunctions and to certain point valued nonexpansive maps. The result is used to study best approximation and to show the space of all nonexpansive maps of a bounded hyperconvex space is hyperconvex.
\end{abstract}

\section{INTRODUCTION}

In this paper we will obtain fixed point theorems for certain multifunctions. A multifunction on $D$ is a function with values in the collection of nonempty subsets of its "range" space. A point $x$ of $D$ is stationary or fixed point for $M$ if $x$ is contained in the set $M(x)$. Apart from any intrinsic interest multis may have, they are of some importance in economics, game theory and differential inclusions [4 and 5]. They appear to be a necessary ingredient for the understanding of nonlinear one parameter semigroups [3].

Perhaps the first multi fixed point theorem was Kakutani's generalization of Brouwer's Theorem. If $M$ is defined on a Euclidean ball $B$, has nonempty closed convex values, and has "graph", $\bigcup\{(x, M(x)): x \in B\}$, which is closed in $B \times B$, then $M$ has a fixed point. The closed graph assumption in Kakutani's Theorem is equivalent to upper semicontinuity of the multi in the Hausdorff metric. If a stronger assumption is made, namely continuity in the Hausdorff metric, a very easy proof is possible. For each $x$ in $D$ let $T(x)$ be the unique point of $M(x)$ which is closest to $x$. Hausdorff continuity of $M$ implies that the point map $T$ is a continuous self map of $D$ so Brouwer's Theorem gives the result under this stronger assumption. Here our fixed point theorem will be established by the same selection trick.

Recall that for bounded sets $A$ and $B$ in a metric space, $S$, we set

$$
H_{0}(A, B)=\sup \{\operatorname{dist}(x, B): x \in A\}
$$

and then define the Hausdorff metric by

$$
H(A, B)=\max \left\{H_{0}(A, B), H_{0}(B, A)\right\} .
$$

Received by the editors April 1, 1988.

1980 Mathematics Subject Classification (1985 Revision). Primary 47H09, 47H10, 54H25, 54E40, 54G05, 06A23.

Key words and phrases. Multifunction, selection, fixed point, retract, best approximation, nonexpansive, hyperconvex. 
Then a multi is nonexpansive in the Hausdorff metric if

$$
H(M(x), M(y)) \leq \operatorname{dist}(x, y) .
$$

From this point on we will use $d(\cdot, \cdot)$ for both the metric in $S$ and the Hausdorff metric on bounded subsets of $S$.

We will use $B(p ; r)$ to denote the closed (solid) ball about $p$ with radius $r$ in any metric space. A metric space is called hyperconvex if it has the following property. Let $\left\{p_{\alpha}\right\}$ be any collection of points and $\left\{r_{\alpha}\right\}$ corresponding real numbers. If

$$
d\left(p_{\alpha}, p_{\beta}\right) \leq r_{\alpha}+r_{\beta}
$$

then

$$
\bigcap B\left(p_{\alpha} ; r_{\alpha}\right) \neq \varnothing
$$

The terminology is due to Aronszajn and Panitchpakdi [1] who proved that a hyperconvex space is a nonexpansive retract of any metric space in which it is isometrically embedded. Soardi [14] and the author [12] showed independently that a nonexpansive (point valued) map of a bounded hyperconvex space has fixed points. Both of these expositions were in the concrete setting of function spaces. The reader will find general expositions in Baillon [2], Kirk [8] and the survey of Jawhari, Misane, and Pouzet [7]. In the last reference the exposition is so general that the rader will have to relearn the definition of metric space. A multifunction generalization of this fixed point theorem is the main goal of this paper.

If $H$ is a hyperconvex space let $J=\bigcap B\left(p_{\alpha} ; r_{\alpha}\right)$ be a nonempty ball intersection. Then $J$ is itself a hyperconvex space. If we define the $\varepsilon$-parallel set $J+\varepsilon$ by

$$
J+\varepsilon=\bigcup\{B(s ; \varepsilon): S \in J\}
$$

then a straightforward application of hyperconvexity yields

$$
J+\varepsilon=\bigcap B\left(p_{\alpha} ; r_{\alpha}+\varepsilon\right)
$$

so $J+\varepsilon$ is closed and if $d(x, J) \leq \varepsilon$ then $x$ is in $J+\varepsilon$. We omit the arguments which may be found in [13]. A standard hyperconvexity argument is to show that an intersection of balls is nonempty by showing the balls have nonempty intersections in pairs. We will use this argument repeatedly.

\section{SELECTION AND FIXED POINTS}

Throughout, $H$ will denote a hyperconvex space and $S$ an arbitrary metric space. We will consider a multifunction $M$ defined on $S$ with values which are nonempty ball intersections in $H$. Since the values of $M$ are then bounded subsets of $H$ the Hausdorff metric is defined for any pair of values of $M$ and nonexpansiveness makes sense for such a map. 
Theorem 1. Let $M$ be a nonexpansive map of $S$ with values which are nonempty ball intersections in $H$. Then $M$ admits a nonexpansive point valued selection. Proof. Consider the family $\left\{M_{\alpha}\right\}$ of all nonexpansive nonempty ball intersection valued multifunctions with $M_{\alpha}(s)$ contained in $M(s)$. This class is nonempty as $M$ itself certainly belongs. We put a partial order on $\left\{M_{\alpha}\right\}$ by inclusion of the graphs in $S \times H$. Let $M_{0}$ be the intersection of the graphs of a maximal chain in $\left\{M_{\alpha}\right\}$. For each $s$ in $S$ it is clear that $M_{0}(s)$ is a nonempty ball intersection contained in $M(s)$. We need to show that $M_{0}$ is nonexpansive in the Hausdorff metric. For any pair of points $s$ and $t$ in $S$ let $r=d(s, t)$. Then as each $M_{\alpha}$ is nonexpansive we have

$$
M_{0}(s) \subset M_{\alpha}(s) \subset M_{\alpha}(t)+r .
$$

It follows that $M_{0}(s)$ is in $M_{0}(t)+r$ and since the argument is symmetric we have that $M_{0}$ is nonexpansive.

Now we will show the minimality of $M_{0}$ implies that it is point valued. Suppose for some point $t$ in $S$ the value $M_{0}(t)$ contains $w_{1}$ and $w_{2}$. We then define

$$
M^{*}(\cdot)=M_{0}(\cdot) \cap B\left(2_{1} ; d(\cdot, t)\right)
$$

on $S$. We claim that $M^{*}$ is a nonexpansive nonempty ball intersection valued multi with

$$
M^{*}(\cdot) \subset M_{0}(\cdot)
$$

Moreover $M^{*}(t)=\left\{w_{1}\right\}$ so minimality of $M_{0}$ shows that $w_{1}$ and $w_{2}$ could not be distinct points. Thus we are done once we establish our claim.

It is immediate from the definition of $M^{*}$ that the values are ball intersections. To show the values are nonempty set $r=d(t, s)$ yielding

$$
\left\{w_{1}\right\} \subset M_{0}(t) \subset M_{0}(s)+r .
$$

Thus $B\left(w_{1} ; d(s, t)\right)$ meets $M_{0}(s)$ so $M^{*}(s)$ is nonempty.

To show that $M^{*}$ is nonexpansive take a pair of points $s_{1}$ and $s_{2}$ in $S$ and set $r=d\left(s_{1}, s_{2}\right)$. Suppose $c$ is a point of $M^{*}\left(s_{1}\right)$. We must show that there is a point $c^{\prime}$ in $M^{*}\left(s_{2}\right)$ with $d\left(c, c^{\prime}\right) \leq r$. Let $M\left(s_{2}\right)=\bigcap B_{\lambda}$. Thus we must show

$$
\bigcap B_{\lambda} \cap B\left(w_{1} ; d\left(t, s_{2}\right)\right) \cap B(c ; r) \neq \varnothing \text {. }
$$

Now $B(c ; r) \cap B_{\lambda} \neq \varnothing$ because $M_{0}$ was nonexpansive. Next we claim

$$
B\left(w_{1} ; d\left(t, s_{2}\right)\right) \cap B_{\lambda} \neq \varnothing
$$

for we showed above that $M^{*}$ had nonempty values. Finally we claim

$$
B\left(w_{1} ; d\left(t, s_{2}\right)\right) \cap B(c ; r) \neq \varnothing
$$

for

$$
\begin{aligned}
d\left(w_{1}, c\right) & \leq d\left(t, s_{1}\right) \leq d\left(t, s_{2}\right)+d\left(s_{2}, s_{1}\right) \\
& =d\left(t, s_{2}\right)+r
\end{aligned}
$$


where the first inequality holds because $c$ is in $M^{*}\left(s_{1}\right)$. Since the intersection of balls is nonempty in pairs, the complete intersection is nonempty and we may take $c^{\prime}$ to be any point of this complete intersection. This completes the proof.

Recall that if $M$ is a multi from $S$ to $S$ then $s$ is a fixed point of $M$ if $S$ is contained in $M(s)$.

Corollary 2. If $M$ is a nonexpansive nonempty ball intersection valued multion a bounded hyperconvex space $H$ then $M$ has fixed points.

Proof. We apply the Selection Theorem to obtain a point valued nonexpansive map $T$. The (point valued) nonexpansive map theorem yields an $x$ in $H$ with $T x=x$. Thus $M(x)$ contains $x$.

Remarks. (1) For both the theorem and the corollary we do not know if nonempty ball intersection values can be replaced with nonempty bounded hyperconvex values. Hyperconvex sets have poor intersection properties. Baillon has shown that a collection of hyperconvex sets in $H$ has a nonempty hyperconvex intersection if each finite subcollection has a nonempty hyperconvex intersection [2].

(2) The analogous properties in Hilbert space may fail. Even in finite dimensions there is a point selection only if the Lipschitz constant of the selection is allowed to increase. Thus in infinite dimensional Hilbert space it is no surprise that there may be no point valued selection for any Lipschitz constant. Examples of this can be found in [5]. The question of whether a nonexpansive multi on a closed bounded convex set in Hilbert space with nonempty closed convex values has fixed points is apparently open.

Besides being nonempty we can show that the fixed point set has some structure.

Corollary 3. Let $M$ be a nonexpansive nonempty ball intersection valued multi on a hyperconvex space $H$ with a nonempty fixed point set. Then $\operatorname{Fix}(T)$ is a hyperconvex set.

Proof. This is really a corollary as the proof of Theorem 1. We will show that the selection of a point valued map can be made preserving the fixed point set of $M$. As before we define $M_{0}$ but now require of the members of the maximal chain that they all have the same fixed point set as $M$. Now we must show that the one point reduction can also be done preserving the fixed point set. If $u$ in $H$ is such that $M_{0}(u)$ contains more than one point and contains a fixed point then $u$ itself is the unique fixed point of $M_{0}(u)$. We set $w_{1}=u$ as in the proof of the theorem. If $x$ is fixed for $M_{0}$ it is trivial that $x$ is in $B\left(w_{1} ; r\right)$ where $r=d(x, u)$. Thus in this case $M^{*}$ will have $\operatorname{Fix}\left(M^{*}\right)$ contained in $\operatorname{Fix}\left(M_{0}\right)$. As the graphs nest downward we actually have equality. In case $M_{0}(u)$ is fixed point free we must select $w_{1}$ in $M_{0}(u)$ with a little care. Suppose $M_{0}(u)=\bigcap B_{\lambda}$ and that $x$ is fixed for $M_{0}$. Then $B(x ; d(x, u))$ 
meets the ball intersection $\cap B_{\lambda}$ as $M_{0}$ is nonexpansive. Thus

$$
\bigcap B(x ; d(x, u)) \cap M_{0}(u) \neq \varnothing
$$

by hyperconvexity where the first intersection is over all fixed points of $M_{0}$. We take $w_{1}$ to be any point in this nonempty intersection and with that choice have our claim that $M$ admits a nonexpansive point selection $T$ with $\operatorname{Fix}(T)=$ Fix $(M)$. It was shown in [11] that the fixed point set of a nonexpansive point valued map of a hyperconvex space is hyperconvex. Then $\operatorname{Fix}(M)=\operatorname{Fix}(T)$ is hyperconvex and we are done.

Remark. In the reference given in the proof above the setting was a function space and the domain was assumed bounded. The boundedness assumption can be dispensed with if $\operatorname{Fix}(T)$ is assumed nonempty. See also [2] for the hyperconvex setting where again the assumption that the domain be bounded can be replaced with the assumption that $\operatorname{Fix}(T)$ is not empty.

Let $M$ be a nonexpansive nonempty ball intersection valued multi on a hyperconvex space $H$. For any $\varepsilon>0$ we define the multi $M_{\varepsilon}$ by $M_{\varepsilon}(x)=$ $M(x)+\varepsilon$ where $M(x)+\varepsilon$ is the $\varepsilon$-parallel set of $M(x)$. Then $M_{\varepsilon}$ is also a nonempty ball intersection valued by properties of parallel sets given in the introduction. We claim that $M_{\varepsilon}$ is nonexpansive. Here is the argument for this claim. Suppose $d(x, y)=r$ and $c$ is a point in $M(x)+\varepsilon$. Also suppose $M(y)=\bigcap B\left(x_{\alpha} ; r_{\alpha}\right)$. Then for some $s$ in $M(x)$ we have $d(c, s) \leq \varepsilon$ and also, as $M$ is nonexpansive, there is a point $s^{\prime}$ in $M(y)$ so that $d\left(s, s^{\prime}\right) \leq r$. Then

$$
d\left(c, x_{\alpha}\right) \leq d(c, s)+d\left(s, s^{\prime}\right)+d\left(s^{\prime}, x_{\alpha}\right) \leq \varepsilon+r+r_{\alpha} .
$$

Thus there is a point $c^{\prime}$ in $M_{\varepsilon}(y)$ with $d\left(c, c^{\prime}\right) \leq r$.

Definition. The $\varepsilon$-fixed point set of a multi $M$ is given by

$$
\operatorname{Fix}_{\varepsilon}(M)=\{x \in H: d(x, M(x)) \leq \varepsilon\} .
$$

Corollary 4. Let $M$ be a nonexpansive nonempty ball intersection valued on $H$. Then the $\varepsilon$-fixed point is hyperconvex.

Proof. The $\varepsilon$-fixed point set of $M$ is the fixed point set of $M_{\varepsilon}$.

Remark. It was shown in [13] that the $\varepsilon$-fixed point set of nonexpansive point valued map of a hyperconvex space is hyperconvex by exploiting the existence of certain retract maps which move points small distances.

If $A$ is any nonempty bounded set in $H$ we define the ball hull of $A$ by

$$
b(A)=\bigcap\left\{B_{\alpha}: B_{\alpha} \supset A\right\}
$$

where $\left\{B_{\alpha}\right\}$ here is the collection of all balls in $H$. If $M$ is a nonexpansive multi with nonempty bounded values we define the map $b M$ by

$$
(b M)(x)=b(M(x)) \text {. }
$$

It is easily seen that then $b M$ is nonempty ball intersection valued. It is less clear that $b M$ is also nonexpansive. We argue as follows. Let $c$ be in 
$b M(x), d(x, y)=r$, and $b M(y)=\bigcap B\left(x_{\alpha} ; r_{\alpha}\right)$. For each $\alpha$ it is easy to show that $M(x)$ is contained in $B\left(x_{\alpha} ; r_{\alpha}+r\right)$. Thus $c$ being in the ball hull of $M(x)$ is in this ball as well. Hence $d\left(c, x_{\alpha}\right) \leq r_{\alpha}+r$ and so

$$
B(x ; r) \cap B\left(x_{\alpha} ; r_{\alpha}\right) \neq \varnothing .
$$

It follows that $B(c ; r)$ meets $b M(y)$. This gives us a point $c^{\prime}$ in $b M(y)$ with $d\left(c, c^{\prime}\right) \leq r$ so $b M$ is nonexpansive.

Corollary 5. If $M$ is nonexpansive with nonempty values on a bounded hyperconvex space $H$ then there exists a point $x$ with $x$ contained in $b M(x)$.

Proof. Immediate from the Selection Theorem and the preceding discussion.

If $A$ is any bounded nonempty set in $H$ we set $r(A)=(1 / 2) \operatorname{dia}(A)$ and define the (unrestricted) center of $A$ to be the set

$$
Z A=\bigcap\{B(x ; r(A)): x \in A\} .
$$

Then $Z A$ is a nonempty ball intersection. If $z$ is in $Z A$ then $A$ is contained in $B(z ; r(A))$ and conversely if $z$ has this property then $z$ is in $Z A$. So in a hyperconvex space we see that the (Chebyshev) radius is half of the diameter. It is a direct application of hyperconvexity that $r(b A)=r(A)$ and $Z b A=Z A$. A good reference on Chebyshev points and approximation is [6].

If $M$ is a multi on $H$ with bounded nonempty values we define the multi $Z M$ by

$$
(Z M)(x)=Z(M(x)) .
$$

This is not necessarily nonexpansive even if $M$ is. (We give examples later in this section.) In spite of this fact we have

Theorem 6. If $M$ is a nonexpansive multi with nonempty bounded values on a hyperconvex space $H$ then there is a nonexpansive point valued selection from $Z M$.

Proof. We can embed $H$ isometrically in $C(E)$ for some extremely disconnected compact Hausdorff space $E$. Such a function space is itself hyperconvex. See [7 and 9] for these facts. Let $M^{\prime}=b M$ where the centers are computed in $C(E)$. For each $x$ in $H$ the set $M^{\prime}(x)$ is a bounded closed order interval in $C(E)$ and $M^{\prime}$ is nonexpansive from $H$ into $C(E)$. Hence

$$
M^{\prime}(x)=\left\{f \in C(E): l_{x} \leq f \leq u_{x}\right\}
$$

where $l_{x}$ and $u_{x}$ are the lower and upper function of bounded closed order interval $M^{\prime}(x)$. These functions are in $C(E)$ even if they are not in $H$. The map $S$ defined by $S x=\frac{1}{2}\left(l_{x}+u_{x}\right)$ is easily shown to be a nonexpansive point valued map of $H$ into $C(E)$. This is still computing centers in $C(E)$ not $H$ however. Now $H$ is a hyperconvex subset of $C(E)$ so there is a retract map $\pi$ of $C(E)$ onto $H$. Then $x \rightarrow \pi S x$ is nonexpansive and point valued from $H$ to $H$. Since the approximation properties will, if anything, be better from $C(E)$ than from $H$ we see that for each $x$ the point $\pi S x$ is in the center of $M(x)$ computed with respect to $H$. So this last map gives the desired selection. 
Corollary 7. Let $M$ be a nonexpansive multi with nonempty values on a bounded hyperconvex space $H$. Then there is a point $x$ with $x$ contained in $Z M(x)$.

Proof. Immediate from the preceding theorem and the hyperconvex fixed point theorem.

For an arbitrary bounded nonempty set $A$ in $H$ the set of centers, $Z A$, need not meet $A$. If $A$ is hyperconvex we do have $Z A \cap A \neq \varnothing$. The intersection $A Z \cap A$ is just what we would get computing the center of $A$ with respect to the hyperconvex superset $A$. We will use the notation $R Z A$ for this restricted center set. Let $M$ be a nonexpansive nonempty hyperconvex valued multi on a bounded hyperconvex space $H$. If $R Z M$ admitted a nonexpansive point valued extension then we would have a fixed point theorem for such multifunctions. Such a selection theorem is not possible as our next example shows.

Example 8. On $[0,1]$ let

$$
l(x)=-1+x \quad \text { and } \quad u(x)=1-x .
$$

We will take for $H$ the set $\left\{f \in L_{\infty}[0,1]: l \leq f \leq u\right\}$. Let $A(\lambda)$ be the set of lines of slope $\lambda$ truncated by $l$ below and $u$ above. Then $H$ is hyperconvex and for each $-1 \leq \lambda \leq 1$ the set $A(\lambda)$ is isometric to the real interval $[-1,1]$ so is also hyperconvex. An easy computation shows that $d(A(1), A(-1))=2 / 3$. For either of these two sets if $z$ is a restricted center we must show $z(0)=0$. This implies that the restricted center of either set is a singleton. From this fact we can compute without difficulty $d(R Z A(1), R Z A(-1))=1$. Similarly the map from $-1 \leq \lambda \leq 1$ to $A(\lambda)$ is nonexpansive. The set $H$ contains isometric copies of the interval $[-1,1]$. Now any set $L$ in $H$ which is an isometric copy of $[-1,1]$ will be hyperconvex so will be the range of a nonexpansive (point valued) retract map on $H$. Putting this retract map together with the map of $L$ to $\{A(\lambda)\}$ obtained by identification gives $M$ a hyperconvex valued multi on $H$. The computation made for $A(1)$ and $A(-1)$ shows that no nonexpansive selection from $R Z M$ exists.

Example 9. We will construct a multi whose values are nonempty order intervals of $R^{2}$. Now an order interval in $R^{2}$ is just a (closed bounded) rectangle so is described by two points, the southwest and the northeast corners. Let $A=$ $\operatorname{Rect}\left[l_{A}, u_{A}\right]$ where $l_{A}$ and $u_{A}$ are the two points in $R^{2}$ given by

$$
l_{A}=(0,0), \quad u_{A}=(10,1) .
$$

Let $B=\operatorname{Rect}\left[l_{B} ; u_{B}\right]$ with

$$
l_{B}=(0,3), \quad u_{B}=(8,3) .
$$

A straightforward and dull computation shows that $d(A, B)=2$ while

$$
d(Z A, Z B)=3 \text {. }
$$

Here is how to embed $A$ and $B$ as values of a multi. In $\S 3$ we will show that the collection of all nonempty ball intersections in a bounded hyperconvex 
space is hyperconvex. So we pick $p$ and $q$ with $d(p, q)=2$. Define $M$ on the doubleton set $\{p, q\}$ by

$$
M(p)=A, \quad M(q)=B .
$$

The Aronszajn-Panitchpakdi Extension Theorem then gives us a nonexpansive rectangle valued map from $\operatorname{Rect}[(0,0) ;(10,10)]$. The assignment of values at $p$ and $q$ implies that $Z M$ fails to be nonexpansive.

It remains open whether a nonexpansive nonempty hyperconvex valued multifunction on a bounded hyperconvex space must have fixed points. These examples show that some otherwise promising downward induction arguments cannot work and make the existence of fixed points less likely.

\section{BEST APPROXIMATION}

In a strictly convex Banach space the best approximation map to a compact convex set is point valued. In other Banach spaces the best approximation map is set valued. We will consider best approximation to ball intersections in a hyperconvex space. If $J$ is a nonempty ball intersection in $H$ and $x$ is an arbitrary point of $H$ it follows immediately from hyperconvexity that the best approximation

$$
A(x)=B(x ; r) \cap J
$$

where $r=d(x, J)$ is a nonempty ball intersection. But easy examples show that $A$ fails to be nonexpansive. For this let $J$ be the ball about the origin in $R^{2}$ of radius 1 ( $l_{\infty}$ metric!). Let $p$ be $(2,0)$ and $q$ be $(1,1)$.

Then $d(p, q)=1$ but $d(A(p), A(q))=2$. In spite of this we have

Theorem 10. Let $J$ be a nonempty ball intersection in $H$ and $A$ the best approximation map. Then there is a nonexpansive nonempty ball intersection valued multi $C$ with $C(x) \subset A(x)$.

Proof. We define $C$ by

$$
C(x)=\bigcap\{A(y)+d(x, y): y \in H\} .
$$

The inclusion $C(x) \subset A(x)$ is clear as is the fact that the values of $C$ are ball intersections. We must show that the values are nonempty and $C$ is nonexpansive. Suppose $J=\bigcap B_{\lambda}$. Then clearly

$$
A(y)=B(y ; d(y, J)) \cap J
$$

is nonempty. So to show $C(x)$ nonempty it suffices to show

$$
B\left(y_{1} ; d\left(y_{1}, J\right)\right)+d\left(x, y_{1}\right) \cap B\left(y_{2} ; d\left(y_{2}, J\right)\right)+d\left(x, y_{2}\right) \neq \varnothing .
$$

But $x$ belongs to both of these sets so $C(x) \neq \varnothing$. For nonexpansiveness pick $x_{1}$ and $x_{2}$ in $H$. We need to show

$$
C\left(x_{1}\right) \subset C^{\prime}\left(x_{2}\right)+d\left(x_{1}, x_{2}\right) .
$$


Thus we need to show

$$
\bigcap\left[A(z)+d\left(x_{1}, z\right)\right] \subseteq \bigcap\left[A(y)+d\left(y, x_{2}\right)+d\left(x_{1}, x_{2}\right)\right] .
$$

But $d\left(y, x_{2}\right)+d\left(x_{1}, x_{2}\right) \geq d\left(y, x_{1}\right)$ so we will be done if we show that the lefthand side of the inclusion is contained in $A(y)+d\left(y, x_{1}\right)$. Take the $z$ value in the left-hand side to be $y$ and the required inclusion is obvious. So we are done.

Corollary 11. Let $J$ be a nonempty ball intersection in a hyperconvex space $H$. Then there is a nonexpansive point valued selection from the best approximation map.

Proof. Since the multi $C$ of Theorem 10 is nonexpansive nonempty ball intersection valued we can apply Theorem 1 .

Remarks. (1) This corollary sharpens a result of [13] which gave a point valued nonexpansive retract map from $J+\varepsilon$ to $J$ which is $\varepsilon$-constant.

(2) The analogous result holds trivially in Hilbert space. There the best approximation map to a compact convex set (which is itself point valued) is nonexpansive.

(3) A standard concrete example is $J=[l \leq g \leq u]$, a nonempty closed bounded order interval in $l_{\infty}$. In this case the existence of the nonexpansive selection of best approximation is completely trivial. For any three real numbers $\{a, b, c\}$ let $\operatorname{Mid}[a, b, c]$ denote the middle value. Then the map

$$
T f(x)=\operatorname{Mid}[l(x), f(x), u(x)]
$$

is the required nonexpansive best approximation selection. In this setting Theorem 1 has an easy proof as well. If $M x=\left[l_{x}, u_{x}\right]$ we set

$$
T(x)=\frac{1}{2}\left(l_{x}+u_{x}\right)
$$

to get a nonexpansive point valued selection from $M$.

(4) The range of a nonexpansive point valued selection of best approximation need not be a ball intersection. The diagonal of the unit square in $R^{2}$ is hyperconvex and has a point valued best approximation defined on the unit square. This set is clearly not a ball intersection. Still not every hyperconvex set will admit a point valued selection of best approximation. For this let the set be the graph of $y=\frac{1}{2} x$ contained in $[0 \leq x \leq 2] \times[0 \leq y \leq 1]$.

Corollary 12. Let $J$ be a nonempty ball intersection in $H$. Let $T$ be a nonexpansive point valued map of $J$ into $H$. Then there is a point $x_{0}$ in $J$ so that $x_{0}$ is a best approximation from $J$ to $T x_{0}$.

Proof. Let $\pi$ be a nonexpansive point valued selection from the best approximation map to $J$. Consider the map $S=\pi T$ from $J$ to $J$. As $S$ is nonexpansive on $J$ there is a point $x_{0}$ in $J$ with $S x_{0}=x_{0}$. This $x_{0}$ is a best approximation in $J$ to $T x_{0}$.

Remark. This is a hyperconvex version of a Theorem of Ky Fan. 
Corollary 13. Let $J$ be a nonempty ball intersection in $H$ and $T$ a nonexpansive point valued map of $J$ into $H$. We assume the boundary condition that for $x$ in $\partial J$ then $x$ is not in the best approximation set from $J$ of $T x$. Then $T$ has a fixed point (which is then necessarily in the interior of $J$ ).

Proof. This follows directly from the preceding corollary.

We have a multifunction version of this also.

Corollary 14. Let $J$ be a nonempty ball intersection in $H$ and $M$ a nonexpansive nonempty ball intersection valued multi on $J$ into $H$. Assume the boundary condition that if $x$ is in $\partial J$ then $x$ is not in the best approximation set of $w$ for any $w$ in $M(x)$. Then $M$ has a fuxed point (necessarily in the interior of $J)$.

Proof. We first take a nonexpansive point valued selection $T$ from $M$. The previous corollary applied to $T$ gives the conclusion.

\section{ULTRA STRUCTURE FOR HYPERCONVEX SPACES}

Let $B I(H)$ denote the collection of nonempty ball intersections in $H$. This is a metric space under the Hausdorff metric as all members are bounded sets.

Theorem 15. $B I(H)$ is hyperconvex.

Proof. Given a collection $\left\{A_{\alpha}\right\}$ of members of $B I(H)$ and corresponding reals $\left\{r_{\alpha}\right\}$ satisfying

$$
d\left(A_{\alpha}, A_{\beta}\right) \leq r_{\alpha}+r_{\beta} .
$$

We must show that

$$
\bigcap B\left(A_{\alpha} ; r_{\alpha}\right) \neq \varnothing \text {. }
$$

Let $A_{\alpha}=\bigcap B(p(\alpha, i) ; r(\alpha, i))$ and consider the subset of $H$ given by

$$
C=\bigcap_{\alpha} B\left(p(\alpha, i) ; r(\alpha, i)+r_{\alpha}\right) .
$$

We claim that this ball intersection is nonempty so is in $B I(H)$ and satisfies certain inequalities in the Hausdorff metric so will be in the required $B I(H)$ ball intersection. To show $C$ is nonempty it is sufficient to consider a pair of balls, $B\left(p(\alpha, i) ; r(\alpha, i)+r_{\alpha}\right)$ and $B\left(p(\beta, j) ; r(\beta, j)+r_{\beta}\right)$. Pick any points $s_{\alpha}$ in $A_{\alpha}$ and $s_{\beta}$ in $A_{\beta}$ with $d\left(s_{\alpha}, s_{\beta}\right) \leq r_{\alpha}+r_{\beta}$ which we may do by the assumptions. Now

$$
\begin{aligned}
d(p(\alpha, i), p(\beta, j)) & \leq d\left(p(\alpha, i), s_{\alpha}\right)+d\left(s_{\alpha}, s_{\beta}\right)+d\left(s_{\beta}, p(\beta, j)\right) \\
& \leq r(\alpha, i)+r_{\alpha}+r_{\beta}+r(\beta, j) .
\end{aligned}
$$

This shows that $C$ is nonempty. Now we must show $d\left(A_{\alpha}, C\right) \leq r_{\alpha}$. If $c$ is in $C$ then for any fixed value of $\alpha$ we claim there is a point $c_{\alpha}$ in $A_{\alpha}$ with $d\left(c, c_{\alpha}\right) \leq r_{\alpha}$. Because $c$ is in $C$ we have $d(c, p(\alpha, i)) \leq r(\alpha, i)+r_{\alpha}$ which implies

$$
B\left(c, r_{\alpha}\right) \cap A_{\alpha} \neq \varnothing \text {. }
$$


This shows that we have the required $c_{\alpha}$. For the other needed inclusion we suppose $c_{\alpha}$ is a point of $A_{\alpha}$ and we must find $c$ in $C$ with $d\left(c, c_{\alpha}\right) \leq r_{\alpha}$. Now $c_{\alpha}$ in $A_{\alpha}$ implies $d\left(c_{\alpha}, p(\alpha, i)\right) \leq r(\alpha, i)$. For any $\beta$ we find $c_{\beta}$ in $A_{\beta}$ so that

$$
d\left(c_{\alpha}, c_{\beta}\right) \leq r_{\alpha}+r_{\beta}
$$

Then

$$
\begin{aligned}
d\left(c_{\alpha}, p(\beta, j)\right) & \leq d\left(c_{\alpha}, c_{\beta}\right)+d\left(c_{\beta}, p(\beta, j)\right) \\
& \leq r_{\alpha}+r_{\beta}+r(\beta, j) .
\end{aligned}
$$

This gives

$$
B\left(c_{\alpha} ; r_{\alpha}\right) \cap C \neq \varnothing
$$

which shows the existence of the required point $c$. Thus we are done.

Let $S$ be an arbitrary metric space and $H$ a bounded hyperconvex space. We will denote the collection of all nonexpansive maps from $S$ into $H$ by $N(S, H)$. If we use the supremum over $S$ then $N(S, H)$ is a metric space.

Theorem 16. $N(S, H)$ is hyperconvex.

Proof. We assume that we have a collection $\left\{T_{\alpha}\right\}$ of members of $N(S, H)$ and a corresponding set of reals $\left\{r_{\alpha}\right\}$ satisfying

$$
d\left(T_{\alpha}(s), T_{\beta}(s)\right) \leq r_{\alpha}+r_{\beta}
$$

for all $s$ in $S$. We define a multi $M$ mapping $S$ into $H$ by

$$
M(s)=\bigcap B\left(T_{s}(s) ; r_{\alpha}\right) .
$$

The hyperconvexity of $H$ shows that for each $s$ in $S$ the value $M(s)$ is a nonempty ball intersection in $H$ by a now familiar argument. To show that $M$ is nonexpansive we take $s_{1}$ and $s_{2}$ in $S$ with $d\left(s_{1}, s_{2}\right)=r$. Let $c_{1}$ be in $M\left(s_{1}\right)$. We will show that there is a point $c_{2}$ in $M\left(s_{2}\right)$ with $d\left(c_{1}, c_{2}\right)<r$. We claim that

$$
B\left(c_{1} ; r\right) \cap M\left(s_{2}\right) \neq \varnothing \text {. }
$$

Now $d\left(c_{1}, T_{\alpha}\left(s_{1}\right)\right) \leq r_{\alpha}$ and $d\left(T_{\alpha}\left(s_{1}\right), T_{\alpha}\left(s_{2}\right)\right) \leq r$ so

$$
d\left(c_{1}, T_{\alpha}\left(s_{2}\right)\right) \leq r+r_{\alpha} .
$$

Thus $M$ is nonexpansive. The Section Theorem gives a point valued nonexpansive map which lies in the $N(S, H)$ ball intersection as required. This finished the proof.

Next we will consider the case that $H$ is contained in $S$. We will denote the set of all nonexpansive maps of $S$ into $H$ which are the identity on $H$ by $N_{0}(S, H)$. We still assume that $H$ is a bounded hyperconvex set.

Theorem 17. $N_{0}(S, H)$ is hyperconvex.

Proof. We again start with a collection $\left\{T_{\alpha}\right\}$ of members of $N_{0}(S, H)$ and corresponding reals $\left\{r_{\alpha}\right\}$ with

$$
d\left(T_{\alpha}(s), T_{\beta}(s)\right) \leq r_{\alpha}+r_{\beta} .
$$


Again define a multi $M$ by

$$
M(s)=\bigcap B\left(T_{\alpha}(s) ; r_{\alpha}\right) .
$$

We will extend the identity map on $H$ to a nonexpansive retract map $\pi$ of $S$ onto $H$ with the additional property that $\pi(s)$ is in $M(s)$ for all $s$ in $S$. Assume that $\pi$ is such an extension to $D$ a superset of $H$ and $x$ is not in $D$. We define the target set

$$
\operatorname{targ}(x)=\bigcap B(\pi w ; d(w, x)) \cap M(x)
$$

with the intersection taken over all $w$ in $D$. Standard binary ball intersection arguments show that $\operatorname{targ}(x)$ is nonempty in $H$. A moment's reflection shows that taking $\pi(x)$ to be any point in this nonempty subset of $H$ gives us a retract map with the required properties of $D \cup\{x\}$. We then obtain $\pi$ on all of $S$ as the union of the graphs of a maximal chain of extensions. This retract map is in the appropriate ball intersection so we have shown that $N_{0}(S, H)$ is hyperconvex.

Suppose that $T$ is nonexpansive point valued on $H$ and $\operatorname{Fix}(T)$ is nonempty. Then $\operatorname{Fix}(T)$ is a nonexpansive retract of $H$.

Corollary 18. Let $T$ be nonexpansive on a bounded hyperconvex space $H$. Then there is a nonexpansive retract map $\pi$ of $H$ onto $\operatorname{Fix}(T)$ so that $\pi T=T \pi$.

Proof. Note first that for any map into $\operatorname{Fix}(T)$ we have $T \pi=\pi$. Now $N_{0}(H, \operatorname{Fix}(T))$ is hyperconvex and consists of all nonexpansive retract maps of $H$ onto $\operatorname{Fix}(T)$. The map $\pi \rightarrow \pi T$ is a nonexpansive map on the bounded hyperconvex space $N_{0}(H, \operatorname{Fix}(T))$ so this map has a fixed point $\pi_{0}$. Then $\pi_{0}=\pi_{0} T$ and this together with the observation at the beginning of the proof gives a commuting nonexpansive retract map.

Remark. This result was obtained in [10] under the assumption that $\operatorname{Fix}(T)$ be nonempty instead of $H$ bounded. The argument here is completely different.

Acknowledgment. This paper was written while visiting Iowa State University. The author thanks the ISU Mathematics Department for its hospitality and support.

\section{REFERENCES}

1. N. Aronszajn and P. Panitchpakdi, Extensions of uniformly continuous transformations and hyperconvex metric spaces, Pacific J. Math. 6 (1956), 405-439.

2. J.-B. Baillon, Nonexpansive mapping and hyperconvex spaces, Fixed Point Theory and Its Applications, (R. F. Brown, ed.), Contemp. Math., vol. 72, Amer. Math. Soc., Providence, R.I., 1988, pp. 11-19.

3. B. Barbu, Nonlinear semigroups and differential equations in Banach spaces, Editura Acad., Bucuresti, 1976.

4. Kim Border, Fixed point theorems with applications to economics and game theory, Cambridge Univ. Press, 1985.

5. A. Cellina and J.- P. Aubin, Differential inclusions, Springer-Verlag, 1984. 
6. R. B. Holmes, $A$ course on optimization and best approximation, Lecture Notes in Math., vol. $257,1972$.

7. E. Jawhari, D. Misane, and M. Pouzet, Retracts: graphs and ordered sets from the metric point of view, Contemp. Math., vol. 56, Amer. Math. Soc., Providence, R.I., 1986.

8. W. A. Kirk, Fixed point theory for nonexpansive mappings, II, Contemp. Math., vol. 18, Amer. Math. Soc., Providence, R.I., 1983, pp. 121-140.

9. H. E. Lacey, The isometric theory of classical Banach spaces, Springer-Verlag, 1974.

10. M. Lin and R. Sine, On the fixed point set of nonexpansive order preserving maps (to appear).

11. W. O. Ray and R. C. Sine, Nonexpansive mappings with precompact orbits, Fixed Point Theory, Lecture Notes in Math., vol. 886, Springer, 1981.

12. R. C. Sine, On nonlinear contractions in sup norm spaces, Nonlinear Analysis 3 (1979), 885890.

13. __ Hyperconvexity and approximate fixed points (to appear).

14. P. Soardi, Existence of fixed points of nonexpansive mappings in certain Banach lattices, Proc. Amer. Math. Soc. 73 (1979), 25-29.

The author would like to thank the referee for bringing to his attention the following (uncited) references to related material on set-valued nonexpansive mappings and the interplay between approximation theory and fixed point theory, as well as to recent work on hyperconvex spaces.

1. M. A. Khamsi, On the fixed point property in metric spaces (preprint).

2. W. A. Kirk, Nonexpansive mappings in product spaces, set-valued mappings and $k$-uniform rotundity, Proc. Sympos. Pure Math., vol. 45, Part 2, Amer. Math. Soc., Providence, R.I., 1986, pp. 51-64.

3. T. C. Lim, A fixed point theorem for multivalued nonexpansive mappings in a uniformly convex Banach space, Bull. Amer. Math. Soc. 80 (1974), 1123-1126.

4. S. Reich, Approximate selections, best approximations, fixed points and invariant sets, J. Math. Anal. Appl. 62 (1978), 104-113.

5. S. Reich, Integral equations, hyperconvex spaces, and the Hilbert ball, Nonlinear Analysis and Applications, Dekker, New York, 1987, pp. 517-525.

Department of Mathematics, Iowa State University, Ames, Iowa 50011

Current address: Department of Mathematics, University of Rhode Island, Kingston, Rhode Island 02881 